\title{
The development and validation of the Bronchiectasis Health Questionnaire
}

\author{
Arietta Spinou ${ }^{1}$, Richard J. Siegert ${ }^{2}$, Wei-jie Guan ${ }^{3}$, Amit S. Patel $^{4}$, \\ Harry R. Gosker $\mathbb{1}^{5}$, Kai K. Lee ${ }^{4}$, Caroline Elston ${ }^{4}$, Michael R. Loebinger ${ }^{6}$, \\ Robert Wilson ${ }^{6}$, Rachel Garrod ${ }^{7}$ and Surinder S. Birring ${ }^{1}$
}

Affiliations: 'Division of Asthma, Allergy and Lung Biology, Faculty of Life Sciences and Medicine, King's College London, London, UK. ${ }^{2}$ School of Public Health and Psychosocial Studies and School of Rehabilitation and Occupation Studies, Auckland University of Technology, Auckland, New Zealand. ${ }^{3}$ State Key Laboratory of Respiratory Diseases, National Clinical Research Centre for Respiratory Disease, Guangzhou Institute of Respiratory Diseases, The First Affiliated Hospital of Guangzhou Medical University, Guangzhou, Guangdong, China. ${ }^{4}$ Dept of Respiratory Medicine, King's College Hospital, London, UK. ${ }^{5}$ NUTRIM School of Nutrition and Translational Research in Metabolism, Dept of Respiratory Medicine, Maastricht University Medical Centre, Maastricht, The Netherlands. ${ }^{6}$ Host Defence Unit, Royal Brompton Hospital, London, UK. ${ }^{7}$ Dept of Physiotherapy, Faculty of Life Sciences and Medicine, King's College London, London, UK.

Correspondence: Surinder S. Birring, Division of Asthma, Allergy and Lung Biology, King's College London, Denmark Hill, London, SE9 5RS, UK. E-mail: surinder.birringanhs.net

@ERSpublications

The BHQ is a brief, valid measure of health status in patients with bronchiectasis http://ow.ly/ddUJ3089LQV

Cite this article as: Spinou A, Siegert RJ, Guan W-j, et al. The development and validation of the Bronchiectasis Health Questionnaire. Eur Respir J 2017; 49: 1601532 [https://doi.org/10.1183/ 13993003.01532-2016].

ABSTRACT Health-related quality of life or health status is significantly impaired in bronchiectasis. There is a paucity of brief, simple-to-use, disease-specific health status measures. The aim of this study was to develop and validate the Bronchiectasis Health Questionnaire (BHQ), a new health status measure that is brief and generates a single overall score.

Patients with bronchiectasis were recruited from two outpatient clinics, during a clinically stable stage. The development of the questionnaire followed three phases: item generation and item reduction using Rasch analysis, validation, and repeatability testing. The BHQ was translated into 11 languages using standardised methodology.

206 patients with bronchiectasis completed a preliminary 65-item questionnaire. 55 items were removed due to redundancy or poor fit to the Rasch model. The final version of the BHQ consisted of 10 items. Internal consistency was good (Cronbach's $\alpha=0.85$ ). Convergent validity of the BHQ with the St George's Respiratory Questionnaire was high $(\mathrm{r}=-0.82 ; \mathrm{p}<0.001)$ and moderate with lung function (forced expiratory volume in $1 \mathrm{~s} \%$ predicted $\mathrm{r}=-0.27$; $\mathrm{p}=0.001)$. There was a significant association between $\mathrm{BHQ}$ scores and number of exacerbations of bronchiectasis in the last 12 months $(p<0.001)$, hospital admissions $(\mathrm{p}=0.001)$ and computed tomography scan bronchiectasis pulmonary lobe counts $(\mathrm{p}<0.001)$. BHQ scores were significantly worse in patients with sputum bacterial colonisation versus no colonisation $(\mathrm{p}=0.048)$. The BHQ was highly repeatable after 2 weeks (intraclass correlation coefficient 0.89 ).

The BHQ is a brief, valid and repeatable, self-completed health status questionnaire for bronchiectasis that generates a single total score. It can be used in the clinic to assess bronchiectasis from the patient's perspective. 


\section{Introduction}

Bronchiectasis is a persistent or progressive condition that is characterised by dilated and often thick-walled bronchi [1]. Symptoms of bronchiectasis include breathlessness, cough, sputum production and haemoptysis. The severity of bronchiectasis can be assessed with physiological, radiological and composite measures such as the Bronchiectasis Severity Index (BSI) and the FACED score [2, 3]. The patient's perception of the condition is also important $[4,5]$. Health status or health-related quality of life is defined as "the perception of the impact of health on an individual's contentment or satisfaction with life in areas they consider important" [6]. Health status is significantly impaired in bronchiectasis [7, 8]. It can be assessed and quantified with validated questionnaires $[9,10]$. They can be used to improve communication between a patient and their clinician, target therapy to specific areas of health, and evaluate the effectiveness of treatment [11].

A recent systematic review has identified several validated health status questionnaires that can be used in bronchiectasis, such as the disease-specific Quality of Life-Bronchiectasis (QoL-B), and the non-disease-specific St George's Respiratory Questionnaire (SGRQ) and Leicester Cough Questionnaire (LCQ) $[8,10,12-14]$. These questionnaires have limitations of being relatively long, do not generate a total score (QoL-B) and are not disease specific (SGRQ and LCQ) [8]. There has been a trend recently to develop brief health status tools with simple overall scores, so that they are practical for clinical use [15]. The increasing use of item response theory methodology to develop health status questionnaires has facilitated briefer tools. One such method uses Rasch analysis, facilitating the creation of unidimensional scales with interval scaling properties [16]. Examples include CAT (chronic obstructive disease (COPD) assessment tool) and K-BILD (King's Brief Interstitial Lung Disease questionnaire) [15, 17]. We set out to develop and validate a bronchiectasis-specific health status questionnaire. Our aim was to develop a well-validated tool that was brief and easy to administer and interpret, with a single overall score.

\section{Methods}

Phase 1: item generation and participants

Item generation

A preliminary Bronchiectasis Health Questionnaire (BHQ) was developed following: 1) a systematic literature review of health status questionnaires in bronchiectasis and review of other health status measures [8]; 2) face-to-face semistructured interviews with 12 consecutive patients with bronchiectasis; and 3) feedback from a multidisciplinary team consisting of respiratory physicians, respiratory physiotherapists, a nurse, a pharmacist and a sociologist. The aim of the interviews was to identify relevant issues and topics to inform the item generation. The interviews began with open-ended questions and continued with more specific topics. Examples of open-ended questions were: Can you describe what it is like to have bronchiectasis? How does it affect your everyday life? The interviews were conducted until saturation (no new items were emerging) [10]. Patients were asked to complete and comment on a preliminary version of the questionnaire. The items of the questionnaire referred to the last 14 days and responses were selected from a seven-point Likert scale. The response scales for items whose high scores represented poor health status were reversed to ensure high scores represented good health status for consistency of analysis.

\section{Participants}

The preliminary BHQ was evaluated in patients with bronchiectasis attributed to various aetiologies, excluding cystic fibrosis. Consecutive patients were recruited prospectively from secondary care (King's College Hospital, London, UK) and tertiary care (Royal Brompton Hospital, London, UK) specialist clinics from November 2012 to August 2014. Patients reporting an exacerbation of their bronchiectasis in the previous 2 weeks were excluded. A substudy was conducted in an independent group of consecutive patients in a specialist bronchiectasis clinic in Guangzhou (Guangdong, China) to assess the clinical properties of a translated BHQ (patient interviews, internal reliability and construct validity by assessing the relationship with computed tomography (CT) scan affected pulmonary lobe count). Demographics and clinical characteristics were recorded. Patients completed the BHQ, SGRQ and visual analogue scales (VASs, 0$100 \mathrm{~mm}$ ) for severity of breathlessness (dyspnoea), cough and sputum, independently when attending their routine clinical appointment. A researcher who was not involved in the clinical care of the patient

Support statement: A. Spinou's PhD was financially supported by the Greek State Scholarships Foundation (IKY). For the Bronchiectasis Health Questionnaire translations, MAPI Language Services (Lyon, France) was funded by King's College Hospital, Research and Development (London, UK). R. Wilson and M.R. Loebinger were supported by the NIHR Respiratory Biomedical Research Unit at the Royal Brompton NHS Foundation Trust and Imperial College London (London, UK). S.S. Birring was supported by King's College Hospital, Research and Development and King's College London (London, UK).

Conflict of interest: Disclosures can be found alongside this article at erj.ersjournals.com 
administered the questionnaires. Patients were excluded from the study if they could not read the questionnaire or completed $<85 \%$ of the items. The diagnosis of bronchiectasis was the first and main aetiology, and was based on clinical characteristics and CT scan, consistent with the British Thoracic Society guidelines [1]. Sputum colonisation status was recorded using the clinical records of the most recent sputum analyses. Sputum bacteria colonisation was assessed and defined as at least two positive cultures, a minimum 3 months apart and within 1 year [2]. All patients provided written informed consent and the study was approved by the Research Ethics Committee London - Queen Square (12/LO/1437).

\section{Phase 2: item reduction, Rasch analysis and validation}

\section{Item reduction}

Items were removed if there was a significant floor effect or ceiling effect response $\geqslant 50 \%$ [18]. Items with missing values $\geqslant 5 \%$ were eliminated. The weaker items of highly intercorrelated item pairs $(r>0.8)$ were removed by face validity [17].

\section{Rasch analysis}

Rasch analysis assesses the fit of a questionnaire to a unidimensional model [19]. A questionnaire that fits the Rasch model has interval scaling properties. Rasch analysis is based on the theory that the response to an item is governed by two factors: the level of ability of the person and the level of difficulty represented by the item. Patients are more likely to respond to items of lower difficulty (less severe health status) and less likely to respond to more difficult items (more severe health status). Rasch analysis was performed for the development of BHQ and items that did not meet the model were removed. This involved an iterative statistical process where the item with the poorest fit to the model was removed and the remaining items retested until a reliable and unidimensional scale was identified. The item-trait interaction was used to assess the questionnaire fit to a unidimensional model. Items were removed if they did not fit to the model, indicated by a significant Chi-squared test for the overall item-trait interaction or individual items. The summation of the difference between the observed and expected scores for all persons, called item fit residuals $\leqslant-2.5$ or $\geqslant 2.5$, indicated items that did not measure the same attribute as the domain being tested and were also removed. Summary item fit residuals and person fit residuals were item-person interaction statistics transformed to approximate a z-score; mean \pm SD residual of $\sim 0 \pm 1$ indicated good fit to the model. The Person Separation Index (PSI) aimed to assess the ability of the BHQ to discriminate patients with different levels of health status impairment and test the reliability of the fit statistics. Internal consistency was assessed using Cronbach's $\alpha$ coefficient. Likert response scale weightings for individual items were combined if necessary to ensure they detected progressive changes in health status. The BHQ total score was obtained following logit transformation of raw responses and then transformed to a range of $0-100$ (100 indicating best health status). Differential item functioning (DIF) was evaluated to assess the influence of age, sex, aetiology of bronchiectasis, forced expiratory volume in $1 \mathrm{~s}(\mathrm{FEV} 1) \%$ predicted and colonisation status (colonisation, no colonisation). The final version for the BHQ was retested with Rasch analysis.

\section{Construct validity}

Construct (convergent/divergent) validity, an assessment of an instrument against other standards that provide an indication of the true value of measurements, was assessed by investigating the relationship between the final BHQ and lung function, CT scan bronchiectasis pulmonary lobe count, exacerbations, hospitalisations, sputum bacterial colonisation, symptom scales and other health status questionnaires. Spirometry was assessed within 6 months of completion of the BHQ. Patients completed the SGRQ, which is a respiratory-specific health status questionnaire originally developed for patients with COPD and asthma [14]. The SGRQ has been validated in bronchiectasis and has been the most widely used questionnaire in this condition $[8,9]$. Patients also completed three symptom severity VASs $(0-100 \mathrm{~mm})$ for breathlessness (dyspnoea), sputum and cough (timeframe last 2 weeks) [11]. The BHQ was administered first, and the SGRQ and VASs were completed in a random order. All measurements were performed prior to patients seeing the doctor. CT scan bronchiectasis pulmonary lobe counts were assessed by an experienced pulmonary radiologist blinded to clinical details in an independent group of patients in Guangzhou. A CT scan was assessed if available within the last 12 months.

\section{Phase 3: repeatability}

To investigate the repeatability of the BHQ, a subgroup of unselected, consecutive patients completed the BHQ on two occasions, 2 weeks apart. Only patients whose condition was considered clinically stable by both clinician and patient were recruited (patients reporting their condition was unchanged on a global rating of change scale). Patients were excluded if they had an upper or lower tract respiratory infection or change of medication within the previous 2 weeks. 


\section{BHQ translations}

The BHQ was translated into 11 languages by MAPI Language Services (Lyon, France), specialists in health status questionnaire translation: nine Indo-European (Dutch for Belgium and the Netherlands, English for the USA, French for Belgium and France, German, Italian, and Spanish for Spain and the USA), one Japonic (Japanese) and one Sino-Tibetan (Mandarin). In each country, the following translation method (i.e. linguistic validation) was used: 1) in-depth analysis of the original (i.e. concept definition) and suggestion of suitable translation alternatives; 2) forward/backward translation step (or adaptation for English and Spanish versions); 3) review of the back translation by the developer; and 4) review of the translated questionnaire by a respiratory clinician fluent in the language. The clinical properties of a translated BHQ were assessed further in a single centre in China (Mandarin): patient interviews, internal reliability and construct validity (association with CT scan pulmonary lobe scores).

Analysis

SPSS version 22.0 (SPSS, Chicago, IL, USA), Prism 5 (GraphPad, La Jolla, CA, USA) and RUMM2030 (www.rummlab.com.au) were used for statistical analysis. Mean \pm SD was used as descriptive statistics for parametric distributions and median (interquartile range) for nonparametric distributions. Significance was set as $\mathrm{p}<0.05$. Correlations between parameters were assessed with Pearson's $r$ and Spearman's $\rho$. Univariate analysis was performed to identify associations between health status and age, sex, bronchiectasis aetiology and colonisation status. Repeatability of the BHQ was assessed with intraclass correlation coefficients (ICCs) and a Bland-Altman plot. The $95 \%$ limits of agreement were calculated as $1.96 \mathrm{sD}$ of within-subject differences. The D’Agostino and Pearson omnibus normality test was used to assess distribution of data. A minimum completion of eight out of 10 items was necessary to generate a BHQ score. The average item score (rounded) of the remaining items was used for missing items.

\section{Results}

\section{Phase 1: item generation and participant characteristics}

12 patients with bronchiectasis (supplementary table E1) were interviewed to identify common themes and items, and interviews were discontinued when they did not yield new items (supplementary table E2). A preliminary version of the BHQ consisting of 65 items was developed following the item generation process (supplementary table E3). The preliminary items covered topics that included respiratory symptoms, functional capacity, psychological health, medications and treatments, relationships, and social function. The response scale timeframes were developed from multidisciplinary team discussion. The preliminary questionnaire was evaluated in four patients with bronchiectasis to assess item wording and no further changes were made.

The BHQ was administered to 221 patients with bronchiectasis to investigate validity. 15 patients $(7.3 \%)$ completed $<85 \%$ of the questionnaire and were excluded (visually impaired, language difficulty, reasons not recorded). The remaining patients did not report any difficulty in completing the BHQ. Demographic and clinical characteristics of the participants are presented in table 1.

\section{Phase 2: item reduction, Rasch analysis and validation Item reduction and Rasch analysis}

The item reduction process is summarised in figure 1 . Two items were removed due to missing responses $>5 \%, 19$ items due to a significant floor effect and three items due to a ceiling effect. Three items were removed because of high item-item correlation. Rasch analysis removed 26 poor-fitting items. There was no individual item misfit in the remaining items and the item-trait interaction was nonsignificant, indicating good fit to the Rasch model. The summary statistics for the final 10-item version of the BHQ indicated a good fit to the model (table 2 and figure 2). The PSI for BHQ total score was 0.85, suggesting it had good discriminant power to detect differing levels of health impairment. A person-item map indicated that BHQ items detected health status across a wide spectrum of health status severity (supplementary figure E1). There was no influence of age, sex, bronchiectasis aetiology, FEV1 \% pred and colonisation status on the response to items (DIF). The response scales for eight items were disordered and corrected to a progressive scale. The mean \pm SD BHQ score was not significantly different in patients with bronchiectasis with coexisting respiratory comorbidities (asthma, COPD, sarcoidosis, pulmonary fibrosis and lung surgery) (58.3 \pm 11.8 versus those with no respiratory comorbidities $60.1 \pm 11.8$; mean difference $1.9,95 \%$ CI of difference $-1.9-5.6 ; \mathrm{p}=0.323$ ).

\section{Validation}

The mean \pm SD BHQ total score (10 item) was $60.0 \pm 11.9$. The BHQ correlated strongly with the SGRQ (all domains and total score) and VAS Dyspnoea, moderately with VAS Cough and VAS Sputum, and weak-moderately with lung function (table 3). The association between BHQ and SGRQ total score 


\section{TABLE 1 Patient demographics and clinical characteristics}

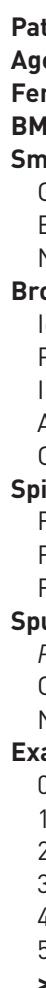

Patients $n$

Female \%

BMI $\mathrm{kg} \cdot \mathrm{m}^{-2}$

Smoking status \%

Current

Ex

Never

Bronchiectasis aetiology \%

Idiopathic

Post-infective

mmunodeficiency

ABPA

Other ${ }^{\#}$

Spirometry

FEV $1 \%$ pred (range)

FVC \% pred

FEV $1 / F V C$

putum colonisation with microorganisms ${ }^{\text {ๆ } \% ~}$

Pseudomonas aeruginosa

Other

41

None

Exacerbations in past 12 months \%

0

1

2

4

$>5$

Hospitalisations in last 12 months \%

$$
\begin{aligned}
& 0 \\
& 1 \\
& 2 \\
& 3 \\
& 4 \\
& 5 \\
& >5
\end{aligned}
$$

\section{Comorbidities ${ }^{+} \%$}

Asthma

Rhinosinusitis

Cardiovascular

COPD

Lung surgery

Diabetes mellitus

Lung fibrosis/sarcoidosis

Malignancy

\section{Respiratory symptoms}

VAS Dyspnoea

VAS Cough

VAS Sputum

SGRQ

SGRQ Total

SGRQ Activities

Data presented as mean \pm SD or median (interquartile range), unless otherwise stated. BMI: body mass index; ABPA: allergic bronchopulmonary aspergillosis; FEV1: forced expiratory volume in $1 \mathrm{~s}$; FVC: forced vital capacity; COPD: chronic obstructive pulmonary disease; VAS: visual analogue scale; SGRQ: St George's Respiratory Questionnaire. \#: other aetiologies (total $n=27$ ) consist of primary ciliary dyskinesia ( $n=10$ ), Kartagener's syndrome ( $n=2)$, inflammatory bowel disease $(n=5)$, rheumatoid arthritis $(n=6)$, Young's syndrome $(n=3)$ and yellow nails syndrome $(n=1)$; " : sputum colonisation status does not add up to $100 \%$ as participants could be chronically infected with $P$. aeruginosa and other microorganisms (sputum bacteria colonisation was assessed and defined as at least two positive cultures, a minimum 3 months apart and within 1 year); ${ }^{+}$: comorbidities do not add up to $100 \%$ as participants could have no comorbidities or more than one comorbidities. 


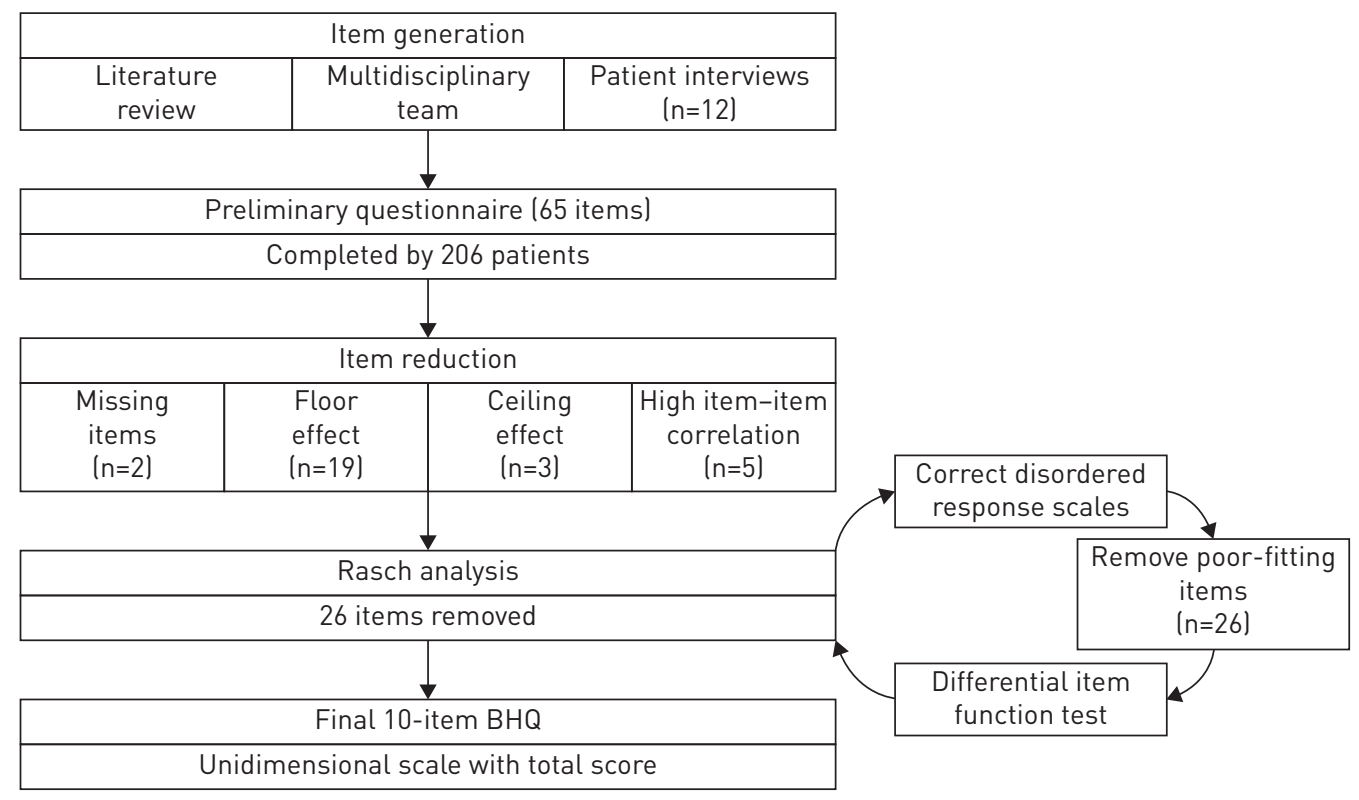

FIGURE 1 Summary of the development of the Bronchiectasis Health Questionnaire (BHQ).

remained unchanged when patients with COPD were excluded $(\mathrm{r}=-0.826 ; \mathrm{p}<0.001)$. There were significant differences in BHQ scores between categories of FEV1 \% pred (ANOVA $\mathrm{p}=0.007)(\mathrm{mean} \pm \mathrm{SD}$ BHQ score for group FEV1 \% pred $<50 \% 55.6 \pm 9.5$ versus $>80 \% 64.1 \pm 11.1$; mean difference $8.5,95 \%$ CI of difference 1.7-15.1; $\mathrm{p}=0.008$ ) (figure 3). There were no significant differences in BHQ scores between FEV $1 \%$ pred $<50 \%$ versus $50-80 \%$ and $>80 \%$ versus $50-80 \%$ ( $>>0.05)$. There were no significant differences in BHQ scores between sex $(p=0.48)$ or bronchiectasis aetiology $(p=0.23)$. BHQ score did not correlate with age $(\mathrm{r}=-0.04 ; \mathrm{p}=0.57)$. The mean \pm SD BHQ score was worse in patients with sputum colonisation (any microorganism) (57.5 \pm 10.2 versus no colonisation $61.1 \pm 12.7$; mean difference $3.6,95 \%$ CI of difference 0.04-7.20; $\mathrm{p}=0.048$ ). The mean \pm SD BHQ score in patients with sputum Pseudomonas aeruginosa colonisation was $57.4 \pm 9.9$ versus no colonisation $61.1 \pm 12.7$ (mean difference $3.7,95 \%$ CI of difference -0.04-7.4; $\mathrm{p}=0.053$ ). BHQ scores were significantly associated with exacerbations and hospitalisation in the last 12 months related to bronchiectasis (table 3, figure 4 and supplementary figure E2). The mean \pm SD BHQ score was lower (worse) in patients reporting exacerbations within the last 12 months (58.8 \pm 11.4 versus no exacerbations $68.4 \pm 11.6$; mean difference $9.6,95 \%$ CI of difference $4.9-14.3 ; \mathrm{p}<0.001$ ) (figure 4). The mean \pm sD BHQ score was lower in patients reporting hospital admissions for bronchiectasis within the last 12 months (54.0 \pm 10.9 versus no hospital admission $61.8 \pm 11.7$; mean difference $7.8,95 \% \mathrm{CI}$ of difference 3.8-11.8; $\mathrm{p}<0.001$ ) (supplementary figure E2). There was a significant association between

TABLE 2 Summary of Rasch analysis

Rasch statistics

BHQ total score

Number of items
Chi-squared fit statistic
Degrees of freedom
p-value
Item fit residual (mean \pm sD)
Person fit residual (mean $\pm s D$ )
PSI
Cronbach's $\alpha$

BHQ: Bronchiectasis Health Questionnaire; PSI: Person Separation Index. The Chi-squared statistic assesses item-trait interaction; nonsignificance indicates a good fit to the Rasch model ( $p>0.05$ ). The mean person and item fit residuals are item-person interaction statistics that are z-transformed; a good fit to the Rasch model is when they approximate to a mean \pm SD of $0 \pm 1$. The PSI assesses how well BHQ discriminates subjects with differing levels of health status. Cronbach's $\alpha$ coefficient is a measure of internal reliability. A PSI and Cronbach's $\alpha$ coefficient $\geqslant 0.7$ is considered acceptable. 


\section{The Bronchiectasis Health Questionnaire $(\mathrm{BHQ})^{(} 2014$}

This questionnaire is designed to assess how bronchiectasis affects your life. Please read each question carefully and answer by SELECTING the response that best applies to you. It is important that you answer all questions as honestly as you can.

\begin{tabular}{|c|c|c|c|c|c|c|}
\hline 1. All of the time & 2. Most of the time & 3. A good bit of the time & 4. Some of the time & 5. A little of the time & 6. Hardly any of the time & 7. None of the time \\
\hline \multicolumn{7}{|c|}{ 2. In the last 14 days, I have been much slower at doing things than other people of my age. } \\
\hline 1. All of the time & 2. Most of the time & 3. A good bit of the time & 4. Some of the time & 5. A little of the time & 6. Hardly any of the time & 7. None of the time \\
\hline \multicolumn{7}{|c|}{ 3. In the last 14 days, I have felt anxious. } \\
\hline 1. All of the time & 2. Most of the time & 3. A good bit of the time & 4. Some of the time & 5. A little of the time & 6. Hardly any of the time & 7. None of the time \\
\hline \multicolumn{7}{|c|}{ 4. In the last 14 days, my chest has felt clear. } \\
\hline 1. All of the time & 2. Most of the time & 3. A good bit of the time & 4. Some of the time & 5. A little of the time & 6. Hardly any of the time & 7. None of the time \\
\hline \multicolumn{7}{|c|}{ 5. In the last 14 days, I have been embarassed because of my phlegm (sputum). } \\
\hline 1. All of the time & 2. Most of the time & 3. A good bit of the time & 4. Some of the time & 5. A little of the time & 6. Hardly any of the time & 7. None of the time \\
\hline \multicolumn{7}{|c|}{ 6. In the last 14 days, I have felt short of breath. } \\
\hline 1. All of the time & 2. Most of the time & 3. A good bit of the time & 4. Some of the time & 5. A little of the time & 6. Hardly any of the time & 7. None of the time \\
\hline \multicolumn{7}{|c|}{ 7. In the last 14 days, my sleep has been disrupted because of my bronchiectasis. } \\
\hline 1. Every night & 2. Most nights & 3. Several nights & 4. Some nights & 5. Occasionally & 6. Rarely & 7. Never \\
\hline \multicolumn{7}{|c|}{ 8. In the last 14 days, I have had coughing fits. } \\
\hline 1. Every day & 2. Most days & 3. Several days & 4. Some days & 5. Occasionally & 6. Rarely & 7. Never \\
\hline \multicolumn{7}{|c|}{ 9. In the last 14 days, my phlegm (sputum) contained blood. } \\
\hline 1. Every time & 2. Most times & 3. Several times & 4. Sometimes & 5. Occasionally & 6. Rarely & 7. Never \\
\hline \multicolumn{7}{|c|}{ 10. In the last 12 months, I have taken antibiotic treatments for a chest infection. } \\
\hline 1. More than five times & 2. Five times & 3. Four times & 4. Three times & 5. Twice & 6. Once & 7. None \\
\hline
\end{tabular}

FIGURE 2 The Bronchiectasis Health Questionnaire (BHQ).

BHQ scores and CT scan bronchiectasis pulmonary lobe count in the substudy group of patients $(\mathrm{n}=56$, supplementary table E4) $(\rho=-0.478 ; \mathrm{p}<0.001)$.

Phase 3: Repeatability

Repeatability was investigated in 32 patients. ICCs were consistent with good repeatability (ICC $0.89,95 \%$ CI 0.77-0.94; $\mathrm{p}<0.01$ ). A Bland-Altman plot of BHQ score repeatability is shown in figure 5.

\section{Translation}

The translation process did not reveal any cultural issues as most of the concepts assessed were cross-culturally relevant. The main difficulties consisted in finding conceptual equivalents to the response choices designed as Likert-type scales in seven points (e.g. "All of the time" to "None of the time"). In all countries, translators ensured that the continuity of time was kept between all versions using either adverbs or synonyms (e.g. "Always" for "All of the time"). Items 2, 4 and 10 raised most discussion across languages for idiomatic or syntactic reasons. Solutions were found in order to respect the structure and

TABLE 3 The relationship between the Bronchiectasis Health Questionnaire (BHQ) and clinical measures ${ }^{\#}$

\begin{tabular}{lrr} 
& BHQ correlation coefficient & p-value \\
\hline FEV 1 pred & 0.266 & 0.001 \\
FVC \% pred & 0.252 & 0.003 \\
Exacerbations in last 12 months & -0.486 & $<0.001$ \\
Hospitalisations in last 12 months $^{\text {\% }}$ & -0.328 & 0.001 \\
SGRQ Total & -0.819 & $<0.001$ \\
SGRQ Activities & -0.704 & $<0.001$ \\
SGRQ Symptoms & -0.710 & $<0.001$ \\
SGRQ Impact $^{+}$ & -0.779 & $<0.001$ \\
VAS Dyspnoea $^{+}$ & -0.703 & $<0.001$ \\
VAS Cough $^{+}$ & -0.613 & $<0.001$ \\
VAS Sputum $^{+}$ & -0.481 & $<0.001$
\end{tabular}

Data are presented as Pearson's $r$ unless, otherwise stated. FEV1: forced expiratory volume in $1 \mathrm{~s}$; FVC: forced vital capacity; SGRQ: St George's Respiratory Questionnaire; VAS: visual analogue scale.

\#: n=206 patients; १: exacerbations and hospitalisations related to bronchiectasis; ${ }^{+}$: Spearman's $\rho$. All p-values $<0.01$. 
FIGURE 3 Mean \pm SD Bronchiectasis Health Questionnaire (BHQ) scores for forced expiratory volume in $1 \mathrm{~s}$ (FEV1) categories.

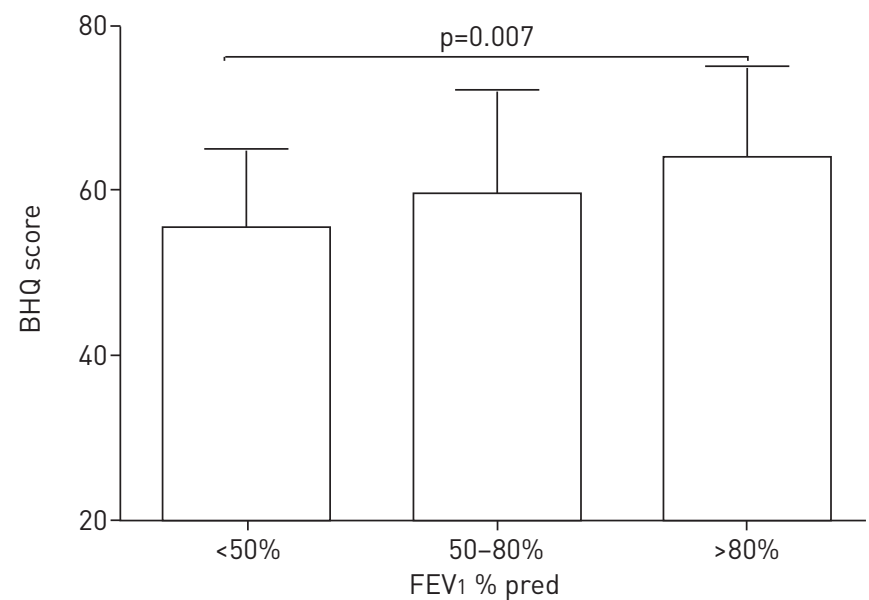

common use of each target language. The clinicians' review proved useful to clarify the concept of "clear chest" in question 4, i.e. meaning "chest clear of sputum or congestion", and led to changes in wording. There were only minor changes to the wording of the Chinese BHQ following patient interviews. Cronbach's $\alpha$ coefficient for the Chinese BHQ (internal reliability) was 0.78 , comparable to the original BHQ. See supplementary Appendix 2 for copies of all translated BHQs.

\section{Discussion}

The BHQ is a health status questionnaire developed and validated specifically for patients with bronchiectasis. The questionnaire items were selected using item response theory to create a unidimensional scale with interval scaling properties. The BHQ is brief, containing just 10 items, and generates a single overall health status score. It is simple to administer and most patients found it easy to complete.

The BHQ items were generated following detailed patient interviews exploring the impact of their lung condition on their health. The preliminary BHQ was assessed using a systematic statistical approach with Rasch analysis, commonly used in the development of health status tools. Items that did not conform to a unidimensional scale were eliminated. Redundant items were also removed, if possible, while retaining essential questionnaire measurement characteristics. Rasch analysis confirmed that the BHQ had good internal scaling of items and was unidimensional. Unidimensional scales are an important attribute of health status questionnaires because they confirm that the instrument measures the intended construct, and allow health status to be quantified as a single number to facilitate clinical utility and interpretation. The convergent validity of the BHQ (association with the SGRQ) was good, suggesting that the removal of redundant items did not compromise the BHQ's ability to measure health status. The BHQ also correlated well with VASs for breathlessness (dyspnoea), and moderately for cough and sputum scales. The internal consistency of the BHQ was good and above the recommended threshold for health status questionnaires [20]. The BHQ scores were worse in patients with frequent exacerbations and hospital admissions in the last 12 months due to their bronchiectasis, and also in those with more extensive disease on CT scan and

FIGURE 4 The association between exacerbations of bronchiectasis and mean \pm SD Bronchiectasis Health Questionnaire (BHQ) scores.

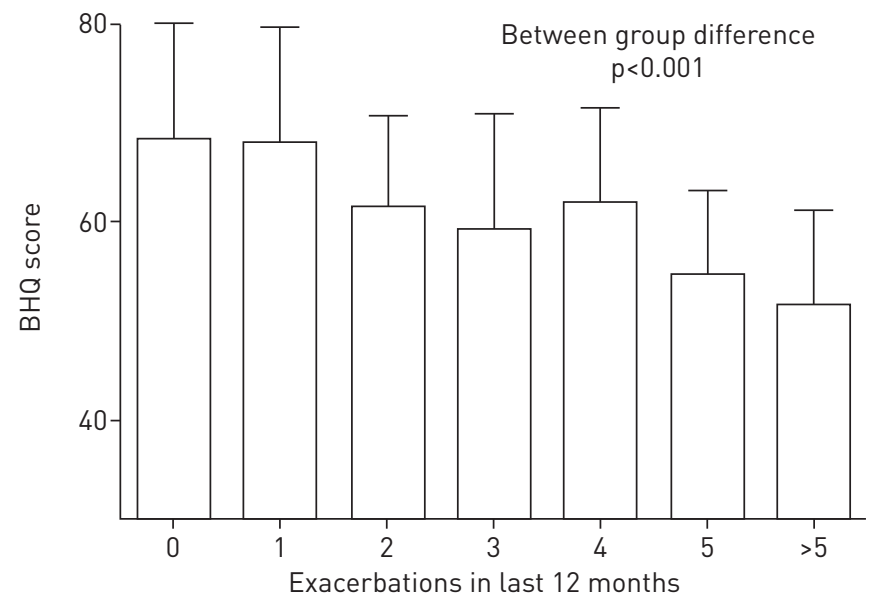


FIGURE 5 Bland-Altman plot (mean $\pm 1.96 \mathrm{sD})$ of repeatability of the Bronchiectasis Health Questionnaire (BHQ).

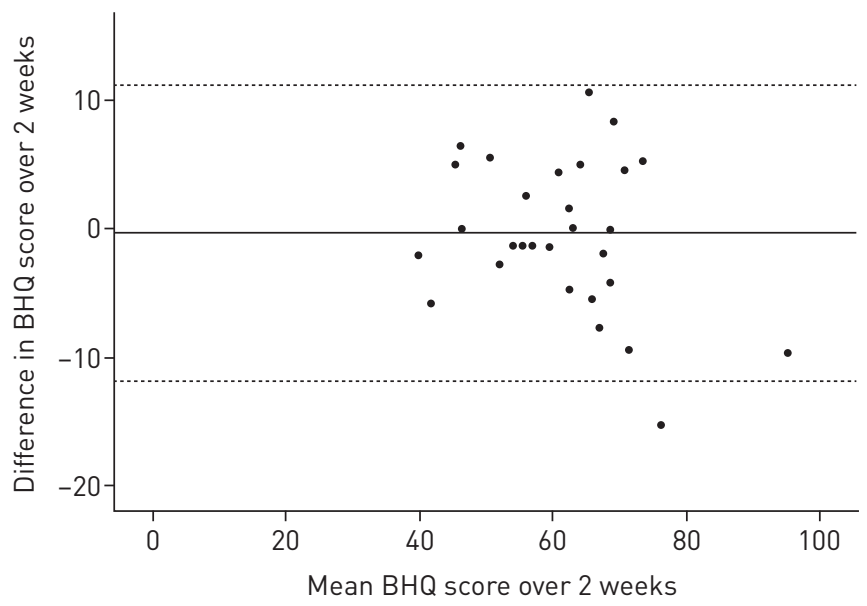

sputum colonisation with microorganisms. The BHQ was highly repeatable over 2 weeks, but further studies are needed to determine this over a longer duration.

The association between health status and lung function (FEV1), although statistically significant, was weak. This is not surprising because health status and measures of lung physiology are very different domains of health. This weak relationship is similar to that reported in other chronic respiratory disorders, such as COPD [21]. A weak association with FEV1 has also been reported for the QoL-B questionnaire [7, 10]. Our findings suggest that health status questionnaires assess a unique aspect of disease severity not captured by objective measures.

The QoL-B is the only other bronchiectasis-specific health status questionnaire that has recently been published and validated $[7,10]$. The QoL-B comprises 37 items and the scores are presented as eight distinct domains. There are several differences between the QoL-B and the BHQ. The QoL-B is a considerably longer questionnaire and does not provide an overall health status score. The BHQ may therefore be more suitable for clinical and research settings where a brief questionnaire is an important practical consideration, and when a single score is desirable for ease of clinical interpretation, such as evaluating the response to therapeutic interventions. In contrast, the QoL-B may be more advantageous when in-depth analysis of health status is required and when there is a need to identify impairment in specific health domains. The questionnaires differ in the methodology used to eliminate items from the preliminary questionnaire to generate the final version. For the QoL-B, item reduction was based on subjective methodology. This may have retained items clinicians and patients consider important, but it is a method that does not select items on their scaling properties. In contrast, the BHQ used Rasch analysis to eliminate items systematically. The validity reported for both questionnaires, such as convergent validity (correlation coefficient with SGRQ: QoL-B -0.38- - 0.85 versus BHQ -0.82), internal consistency (Cronbach's $\alpha$ : QoL-B 0.66-0.94 versus BHQ 0.85 ) and repeatability (ICC: QoL-B $0.67-0.88$ versus BHQ 0.89 ) is generally comparable $[7,8,10,22]$. This suggests that the removal of redundant items to create a very brief tool has not compromised the measurement characteristics of the BHQ. There were some differences in the patient populations in which both questionnaires were validated. The BHQ was validated in an unselected population with bronchiectasis. The QoL-B was validated in clinical trials that selected participants who had cultured Gram-negative sputum organisms [7, 10, 22]. We were unable to compare the performance of the BHQ against the QoL-B as it was not available at the time of this study. Future studies comparing the performance of the BHQ, SGRQ and QoL-B questionnaires are needed to address this limitation, particularly longitudinal assessment, treatment responsiveness and association with disease severity tools such as the BSI.

The SGRQ and LCQ are two other health status questionnaires widely used in the assessment of bronchiectasis $[8,9,23]$. Although they were developed for use in other chronic respiratory conditions, they have been well validated in patients with bronchiectasis. The internal reliability, construct validity and repeatability of the BHQ is comparable to that of the SGRQ and the LCQ $[8,9,24]$. The SGRQ is a considerably longer, comprising of 50 items, and has a greater focus on breathlessness [14]. The LCQ also has a greater number of items $(n=19)$ and its main focus is on health status related to cough [13]. There is overlap in the content of five items between the LCQ and BHQ. The BHQ, however, focuses specifically on symptoms of bronchiectasis, and both breathlessness and cough are represented. A comparative study of the BHQ and LCQ is needed to determine if assessment of health status with the BHQ covers the domains of the LCQ.

We have translated the BHQ into 11 other languages using standardised methodology. The translations were conducted by experts (MAPI Language Services) who are experienced in adapting health status 
questionnaires for use in a wide range of countries. We also sought the input of pulmonary clinicians familiar with bronchiectasis who were literate in these languages. Our translation methodology should be considered the first step in the cultural adaptation of the BHQ. Further independent evaluation of the translated BHQ is necessary to obtain the feedback of patients and also determine its validity, such as the internal consistency. We did assess the validity of the Chinese language BHQ with patient interviews and assessment of construct validity and internal reliability; this was consistent with the original English language $\mathrm{BHQ}$. The publication of a health status questionnaire from the outset in multiple languages may be advantageous in ensuring the standardisation and the quality of the translation (see supplementary Appendix 2 for copies of the translated BHQ in French, Dutch, German, Italian, Spanish, Japanese, Chinese (simplified Mandarin), Belgian (French and Dutch), US Spanish and US English).

There are some limitations with health status questionnaire development methodology. We used Rasch analysis to eliminate items that did not fit a unidimensional model. We eliminated items that were infrequent, redundant or contributed weakly to health status assessment, or did not conform to optimal scaling properties. We may have eliminated items important to patients that may have been retained using methodology based on clinical importance [25]. Our aim was to develop a brief questionnaire that could quantify health status with the least number of items, while retaining validity, so that it was practical for clinical use. Health status questionnaires are not a substitute for identifying health-related issues obtained from a detailed history. The prevalence of idiopathic bronchiectasis in our study was high at $41 \%$. While this may reflect difficultly in identifying causes, the prevalence was similar to $44 \%$ reported in a recent systematic review of 8608 patients [26]. There are a number of well-validated tools now available to assess the severity of bronchiectasis, such as the BSI and FACED tools $[2,3]$. We were unable to retrospectively assess these scores because some of the clinical components of these scores were not available or were significantly out of date. We did not assess the extent of bronchiectasis as the duration between CT scan and study assessments was considerable for many patients. The BSI and FACED tools assess disease severity, which is distinct from health status measures. We did, however, find a significant association between the BHQ and FEV1 and breathlessness, components of both severity tools. To our knowledge, the association between QoL-B and BSI or FACED scores has also not been reported. Further studies should investigate the relationship between the BSI and FACED and health status assessed with the BHQ.

The responsiveness and the minimal clinically important difference (MCID) of the BHQ have not been studied and this needs investigation [27]. The MCID is essential for clinical interpretation of BHQ scores and therefore the BHQ is likely to be limited to a research tool until this is defined. The high PSI for the BHQ was, however, consistent with ability to discriminate differing levels of health status. The sample size of patients $(n=12)$ interviewed was small and it is possible some themes relevant to patients were missed. The sample was small as the interviews were in depth and it was similar to the development of other health status tools such as the LCQ. We were able to generate a large preliminary BHQ questionnaire comprising 65 items that covered a broad range of health domains. It is possible that the presence of comorbidities, such as asthma, COPD, Aspergillus colonisation and immunoglobulin deficiency, may have impacted the assessment of health status. The BHQ items, however, were worded specifically to relate to the patient's bronchiectasis. The patients were recruited from bronchiectasis clinics and bronchiectasis was the primary diagnosis. Our patients had mild physiological impairment and it is possible this may have had an impact on the development of the BHQ. The recruitment of a large sample size $(n=206)$, patients with a wide range of physiological impairment (lowest FEV1 \% pred 18\%) and the lack of DIF with FEV1 suggests this is unlikely. We did not assess the relationship between BHQ scores and the presence of clinically significant nontuberculous mycobacteria; this should be assessed in future. A potential disadvantage of the BHQ is that it does not quantify health status in specific health domains, such as psychological, activity and social. We did, however, set out to develop a very brief and practical health status questionnaire with a single overall score. A detailed assessment of health domains can be achieved with other questionnaires. The purpose of question 10 (number of antibiotics taken for chest or respiratory infections in 12 months) was to capture the impact of frequent respiratory infections on health status. Question 10 may be less responsive when repeated over a shorter time interval. The BHQ does have nine other items that assess over a short interval; hence, the impact of one item is likely to be limited. Patients taking long-term antibiotics may have difficulty in answering question 10. We did not encounter any issues during patient interviews or administration of the questionnaire in a large number of patients; they were able to relate this item to acute infections. The BHQ has been evaluated in only three centres and therefore an external validation, including more overseas centres, is essential in future studies, given the heterogeneous nature of bronchiectasis.

The BHQ has a number of potential applications. It is a quick and valid tool to identify health status issues important to patients in the clinic. It can be used to help formulate shared care plans between the patient and the physician. In summary, the BHQ is brief, easy to administer and well validated. 


\section{Acknowledgements}

The authors would like to thank all patients that participated in this study and the professionals who provided feedback to the BHQ. Natalie Williams (Respiratory Physiologist, King's College Hospital, London, UK) coordinated the translation of the BHQ. We are also grateful to our clinical colleagues who provided expert review of the translated BHQ (Lieven Dupont, University Hospital Leuven, Leuven, Belgium (Dutch/French), Gianluca Cotta, Hospital de la Santa Creu i Sant Pau, Barcelona, Spain (Spanish/Italian), Akio Niimi, Nagoya City University Hospital, Nagoya, Japan (Japanese), and Amadea Heitman, King's College Hospital London, UK and Stefan Kulnik, Kingston University and St George's University of London, London, UK (German)). We also thank colleagues at MAPI Language Services (Lyon, France) who managed the translations (Christelle Tevi, Catherine Acquadro and Axelle Nadjar).

The Bronchiectasis Health Questionnaire (BHQ) 2014 is protected by copyright (@) King's College Hospital NHS Foundation Trust). Reprints of the BHQ and scoring programme are available from the corresponding author.

Author contributions: Conception of work: S.S. Birring; design of work: S.S. Birring, R. Garrod and A. Spinou; participant screening: A. Spinou, K.K. Lee, C. Elston, R. Wilson, M.R. Loebinger and W-j. Guan; study recruitment: A. Spinou and W-j. Guan; data analysis: A. Spinou, R.J. Siegert, A.S. Patel, H.R. Gosker, S.S. Birring and W-j. Guan; drafting manuscript: A. Spinou, S.S. Birring and R. Garrod; revising manuscript: all.

\section{References}

1 Pasteur MC, Bilton D, Hill AT. British Thoracic Society guideline for non-CF bronchiectasis. Thorax 2010; 65 Suppl. 1, i1-i58.

2 Chalmers JD, Goeminne P, Aliberti S, et al. The Bronchiectasis Severity Index. An international derivation and validation study. Am J Respir Crit Care Med 2014; 189: 576-585.

3 Martinez-Garcia MA, de Gracia J, Vendrell Relat M, et al. Multidimensional approach to non-cystic fibrosis bronchiectasis: the FACED score. Eur Respir J 2014; 43: 1357-1367.

4 Brignall K, Jayaraman B, Birring SS. Quality of life and psychosocial aspects of cough. Lung 2008; 186: Suppl. 1, S55-S58.

5 Raj AA, Birring SS. Clinical assessment of chronic cough severity. Pulm Pharmacol Ther 2007; 20: 334-337.

6 Swigris JJ, Kuschner WG, Jacobs SS, et al. Health-related quality of life in patients with idiopathic pulmonary fibrosis: a systematic review. Thorax 2005; 60: 588-594.

7 Quittner AL, O'Donnell AE, Salathe MA, et al. Quality of Life Questionnaire-Bronchiectasis: final psychometric analyses and determination of minimal important difference scores. Thorax 2015; 70: 12-20.

8 Spinou A, Fragkos KC, Lee KK, et al. The validity of health-related quality of life questionnaires in bronchiectasis: a systematic review and meta-analysis. Thorax 2016; 71: 683-694.

9 Wilson CB, Jones PW, O'Leary CJ, et al. Validation of the St. George's Respiratory Questionnaire in bronchiectasis. Am J Respir Crit Care Med 1997; 156: 536-541.

10 Quittner AL, Marciel KK, Salathe MA, et al. A preliminary quality of life questionnaire-bronchiectasis: a patient-reported outcome measure for bronchiectasis. Chest 2014; 146: 437-448.

11 Mutalithas K, Watkin G, Willig B, et al. Improvement in health status following bronchopulmonary hygiene physical therapy in patients with bronchiectasis. Respir Med 2008; 102: 1140-1144.

12 Gao YH, Guan WJ, Xu G, et al. Validation of the Mandarin Chinese version of the Leicester Cough Questionnaire in bronchiectasis. Int J Tuberc Lung Dis 2014; 18: 1431-1437.

13 Birring SS, Prudon B, Carr AJ, et al. Development of a symptom specific health status measure for patients with chronic cough: Leicester Cough Questionnaire (LCQ). Thorax 2003; 58: 339-343.

14 Jones PW, Quirk FH, Baveystock CM, et al. A self-complete measure of health status for chronic airflow limitation. The St. George's Respiratory Questionnaire. Am Rev Respir Dis 1992; 145: 1321-1327.

15 Jones PW, Harding G, Berry P, et al. Development and first validation of the COPD Assessment Test. Eur Respir J 2009; 34: 648-654

16 Patel AS, Siegert RJ, Creamer D, et al. The development and validation of the King's Sarcoidosis Questionnaire for the assessment of health status. Thorax 2013; 68: 57-65.

17 Patel AS, Siegert RJ, Brignall K, et al. The development and validation of the King's Brief Interstitial Lung Disease (K-BILD) health status questionnaire. Thorax 2012; 67: 804-810.

18 Yorke J, Moosavi SH, Shuldham C, et al. Quantification of dyspnoea using descriptors: development and initial testing of the Dyspnoea-12. Thorax 2010; 65: 21-26.

19 Rasch G. Probabilistic Models for Some Intelligence and Attainment Tests. Chicago, University of Chicago Press, 1960

20 Terwee CB, Bot SD, de Boer MR, et al. Quality criteria were proposed for measurement properties of health status questionnaires. J Clin Epidemiol 2007; 60: 34-42.

21 Ketelaars CA, Schlosser MA, Mostert R, et al. Determinants of health-related quality of life in patients with chronic obstructive pulmonary disease. Thorax 1996; 51: 39-43.

22 Olveira C, Olveira G, Espildora F, et al. Validation of a Quality of Life Questionnaire for Bronchiectasis: psychometric analyses of the Spanish QOL-B-V3.0. Qual Life Res 2014; 23: 1279-1292.

23 Birring SS. New concepts in the management of chronic cough. Pulm Pharmacol Ther 2011; 24: 334-338.

24 Murray MP, Turnbull K, MacQuarrie S, et al. Validation of the Leicester Cough Questionnaire in non-cystic fibrosis bronchiectasis. Eur Respir J 2009; 34: 125-131.

25 Patel AS, Siegert RJ, Bajwah S, et al. Rasch analysis and impact factor methods both yield valid and comparable measures of health status in interstitial lung disease. J Clin Epidemiol 2015; 68: 1019-1027.

26 Gao YH, Guan WJ, Liu SX, et al. Aetiology of bronchiectasis in adults: a systematic literature review. Respirology 2016; 21: 1376-1383.

27 Patel AS, Siegert RJ, Keir GJ, et al. The minimal important difference of the King's Brief Interstitial Lung Disease Questionnaire (K-BILD) and forced vital capacity in interstitial lung disease. Respir Med 2013; 107: 1438-1443. 